emotional responses in the writer. Therefore, before embarking on any qualitative research that employs reflective writing, one must keep in mind the risk of causing emotional distress in the writer and proper mitigative measures must be put in place.

\section{Reference}

1. Mohan M. Use of critical reflection as a research method: a case of research-induced distress? Indian J Med Ethics. 2020 Jan-Mar;5(1) NS:1920.DOI: 10.20529/IJME.2020.014.

2. Birch M, Miller T. Inviting intimacy: The interview as therapeutic opportunity. Int J Soc Res Methodol. 2000 Nov; 3(3): 189-202.

3. Campbell R, Adams AE, Wasco S M, Ahrens C E, Sefl T."What has it been like for you to talk with me today?": The impact of participating in interview research on rape survivors. Violence Against Women, 2010 Jan;
16(1):60-83.

4. Cook AS, Bosley G. The experience of participating in bereavement research: Stressful or therapeutic? Death Stud. 1995 Mar-Apr: 19(2), 157-170.

5. Ellis C. Telling secrets, revealing lives relational ethics in research with intimate others. Qual Inq. 2007 Jan; 13(1): 3-29.

6. Koshy K, Limb C, Gundogan B, Whitehurst K, Jafree DJ. Reflective practice in health care and how to reflect effectively. Int J Surg Oncol (N Y). 2017 Jul; 2(6):e20. doi: 10.1097/IJ9.0000000000000020.

7. Medical Council of India.AETCOM - Attitude Ethics and Communication. Competencies for the Indian Medical Graduate. New Delhi: MCl;2018 [cited 2020 Jan 20]. Available from: https://www.mciindia.org/CMS/wpcontent/uploads/2019/01/AETCOM_book.pdf

8. Jasper M A. Using reflective writing within research. J Res Nurs. 2005 May;10(3):247-60.

\title{
Ancillary findings during public health research: A researcher's ethical dilemma
}

\section{NEETHU SURESH}

Keywords: Ancillary findings, anticipating research problems, anonymity, protecting participants, confidentiality

\section{Background}

Sometimes researchers investigating one matter come across other issues in the course of their work. Such findings, not directly related to the subject of research, are called ancillary findings. Researchers encountering ancillary findings may face ethical dilemmas on how to act upon these findings.

\section{The case}

A researcher was investigating the effectiveness of oral health education on the oral hygiene status of 6- to 12-year-old school children studying in government and governmentaided schools in a South Indian state. The researcher conducted focus group discussions with teachers in 16 schools, to understand the dietary habits of children as these can affect their oral health. The participants were promised that the information they provided would be kept confidential and used only for research purposes.

As part of the school meal programme, the government provides lunch and breakfast to school children. School authorities also have the responsibility of providing safe drinking water to the children. During the focus group

Author: Neethu Suresh (neethusuresh@ymail.com), PhD Scholar, Achutha Menon Centre for Health Science Studies, Sree Chitra Tirunal Institute for Medical Sciences and Technology, Thiruvananthapuram, Kerala, 695 011, INDIA.

To cite: Suresh N. Ancillary findings during public health research: a researcher's ethical dilemma. Indian J Med Ethics. 2020 Jan-Mar;5(1) NS: 22-3. DOI: 10.20529/IJME.2020.016.

OIndian Journal of Medical Ethics 2020 discussions with teachers, the researcher found that even though care was taken to provide a healthy breakfast and lunch to the school children, not all schools ensured safe drinking water.

Only one out of the 16 schools was taking adequate steps to provide safe drinking water to children throughout the school hours. In each classroom in that school, boiled water was provided in a pot along with a glass, so that children had access to safe drinking water throughout school hours.

In the remaining schools, children faced difficulties in obtaining safe drinking water. In most schools, boiled water was kept in the kitchen, but in some of these schools the kitchen was locked by $3 \mathrm{pm}$. After $3 \mathrm{pm}$, children depended on tap water or remained thirsty till they reached their homes. In some schools there was a long distance between the classrooms and the kitchen, so children did not have easy access to safe drinking water.

Rural schools which depended entirely on well water faced water shortages in the summers. In these schools, when the power went off they were not able to pump well water. School authorities had therefore instructed children to bring two bottles of water from home, one for drinking and the other for washing their tiffin plates.

The teachers' justified not providing safe drinking water to the children saying that the children could bring water from their homes, and that drinking tap water does not cause any problems; there was not enough fuel to boil water; it was difficult to carry the heavy water-filled jars from the kitchen to the corridor, and there was a water shortage.

Drinking unsafe water can cause many gastrointestinal problems including vomiting and diarrhoea. Moreover, access to safe drinking water is the right of every child. In schools, it 
is the responsibility of the school authorities to ensure that children have access to safe drinking water throughout the school hours.

\section{Questions}

1. What are the ethical issues at stake here?

2. What is the ethical responsibility of the researcher in this situation?
3. Will the researcher's ethical responsibilities be different if she is studying the availability of safe drinking water to school children?

4. What are the researcher's responsibilities when she finds out that ancillary findings contradict government reports?

5. What are the researcher's responsibilities when media reports question the availability of safe drinking water in schools and the media demands a government level enquiry and action?

\section{Unanticipated challenges: Institutional anonymity vs child health}

\section{MANICKAM PONNAIAH}

Keywords: Ancillary / unanticipated findings, public health research, ethical challenges, institutional anonymity

\section{Background}

The case study presented by the researcher reflects on a dilemma faced during her public health research in a setting in South India (1). Her case prompts discussions around the public health context, ethical dilemmas therein, research challenges and relevance to other situations in public health research.

\section{The public health context of the case study}

The ethical dilemma concerning ancillary or incidental findings and the appropriate care options and obligations have been recognised and articulated in the literature. Incidental findings are defined as those concerning an individual research participant that have potential health or reproductive importance, and are discovered during the course of conducting research but are beyond the aims of the study (2). Most of the accounts regarding incidental findings in the literature are about dealing with individual level ancillary findings. In contrast to these, public health research targets communities, populations, institutions and larger geographical areas (3). In such public health studies, ancillary findings do pose ethical dilemmas such as the one faced by the researcher while examining the effectiveness of oral health education on the oral hygiene status of school children within the context of the school health programme in a southern Indian setting (1).

\footnotetext{
Author: Manickam Ponnaiah (manickam@nie.gov.in), Scientist 'E', National Institute of Epidemiology, Ayapakkam, Chennai, 600077 INDIA

To cite: Manickam P. Unanticipated challenges: Institutional anonymity vs child health. Indian J Med Ethics 2020 Jan-Mar;5(1) NS:23-4. DOI: 10.20529/ IJME.2020.017.

Peer Reviewer: Rakhal Gaitonde

CIndian Journal of Medical Ethics 2020
}

\section{Ethical dilemmas highlighted by the case study}

The interesting case study presented by Neethu Suresh (1) highlights ethical dilemmas faced by fellow public health researchers. One such dilemma is maintaining the confidentiality of the institutions studied. In her case, the institutions that did not have the provision of safe drinking water could have been identified. This could be in breach of the confidentiality clause, if stated in the informed consent process.

Further, making such information available in the public domain can give rise to challenges for the schools and further for their community.

A second issue of concern is that of the safety and well-being of the children in the research study and of those similarly situated. It is imperative that safe drinking water is provided and as such the investigator has to find a way to discuss with the teachers the anonymising of the institution's deficiency on that account, and the possibility of communicating it to the relevant departments or stakeholders.

\section{Discussion}

\section{Case study specific challenges}

The case study highlights the methodological challenge of how to mask the identity of study units so that researchers can maintain confidentiality while disclosing their findings for remedial action. In the context of studies based on individuals, it may be possible to consider using masking methods while selecting participants (sampling methods), or assigning interventions (randomisation), or use of a group approach (such as medical camps) for collecting data. However, it is difficult to implement such methods for group or populationbased studies.

The designing of studies to anonymise group level information poses a challenge. However, at the protocol stage, the public health researcher needs to have anticipated that safe drinking water is a variable concerned with the research question 